International Journal of Engineering \&Technology, $7(1.8)(2018) 10-14$
International Journal of Engineering \& Technology
WPC
Website: www.sciencepubco.com/index.php/IJET
Research paper

\title{
An automated exudates detection in diabetic retinopathy fundus images using multi kernel spatial fuzzy c means clustering method
}

\author{
R Ravindraiah ${ }^{1 *}$, S Chandra Mohan Reddy ${ }^{2}$ \\ ${ }^{1}$ Research Scholar, ECE Dept., JNTUA, Anantapuramu, Andhra Pradesh, India \\ ${ }^{2}$ Assoc Professor, ECE Dept., JNTUA College of Engineering, Pulivendula, Andhra Pradesh, India \\ *Corresponding author E-mail: ravindra.ranga@gmail.com
}

\begin{abstract}
Microvasculature change associated with tenacious Hyperglycemia are the hostile effects accompanying to diabetes mellitus. Diabetic Retinopathy (DR) is a progressive complication, which leads to retinal permeability, ischemia, neovascularization and macular edema. The pathology is characterized by variation in capillary diameter, size of microaneurysm, hemorrhage exudates. Thus it stimulates the growth of new abnormal blood vessels so as to nourish the eye muscles. But these newly grown blood vessels are subtle, and may get burst. Therefore it leads to leakage of blood, protein based particles named as exudates. Early determination of the DR signs will help the diabetic patient to eradicate austere vision damage. Medical image processing methods helps the ophthalmologists in easy diagnosis, and to estimate the severity of the pathology. Fuzzy based clustering methods are simple and effective methods that will classify the pathos. This work furnish an improved fuzzy clustering method with induced multi kernel and spatial constraint. Statistical evaluation is done to evaluate the performance of the proposed method.
\end{abstract}

Keywords: Exudates; Diabetic Retinopathy (DR); Fuzzy C Means Clustering algorithm (FCM); Multiple kernel Spatial FCM (MKSFCM).

\section{Introduction}

Exudates are prime pathos of Diabetic Retinopathy (DR) that affect the healthy tissue of eye which leads to vision loss. Swollen, ruptured blood vessels are the characteristics of this pathology. This triggers the generation of new blood vessels within the retina for to nourish the eye tissue. But the newly formed blood vessels are very sensitive and will easily get ruptured, leaking blood, protein based particles [called exudates] into the fundus of eye [1]. This further complicates the situation as it leads to origin of lesions like Micro aneurysms, haemorrhages, soft exudates, hard exudates. Physical distinction of Exudates kills time, highly user reliant and hard to outline the boundaries. Programmed screening systems plays a significant role in Exudate detection. DR is classified as i) Non-Proliferative DR (NPDR) and ii) Proliferative DR (PDR) [2]. NPDR is a replication of clinical hiperpermeabilitas and ineffectual blood vessels triggered by the obstruction and capillary outflow. The exuded blood and protein particles are coagulated as microaneurysms, hemorrhages, cotton wool spots, inter retinal microvascular changes and intravenous complaints [3]. This progresses to advanced or Proliferative DR where blood vessels proliferate. Lack of oxygen to retina further advances the complication that tends to cloud vision and may leads to total retinal damage [4].

This can contribute to deprivation of sight and eventually blindness. DR is generally asymptomatic until it becomes more severe. Many people with mild DR have no symptoms. In more advanced cases, however, blurred vision, floaters or sudden catastrophic loss of vision can occur in one or both eyes. It would be useful to have an automated method of detecting exudates in digital retinal imag- es produced from DR screening programs [5]. Early detection and diagnosis of DR help the patient in preventing severe vision loss. Retinal images obtained by the fundus camera are used to diagnose DR. Automated methods of DR screening help to save time, cost and vision of patients, compared to the manual methods of diagnosis [6][7].

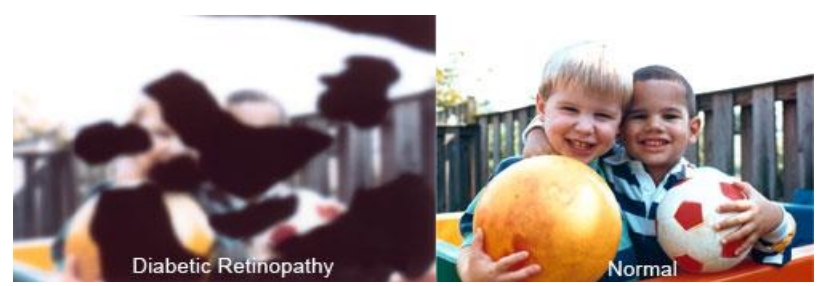

Fig. 1: Vision with and Without Diabetic Retinopathy.

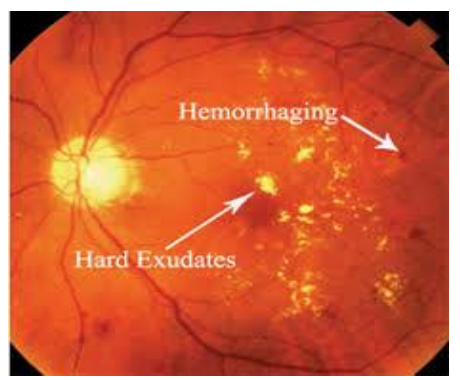

Fig. 2: DR Fundus Image. 


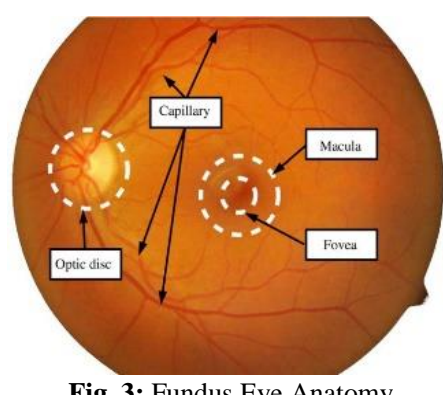

Fig. 3: Fundus Eye Anatomy.

The figure 1 show the impact of DR on vision. It can be observed that the vision consists of black shades which are the resultant of deposited exudates. Figure 2 shows the existence of yellow, red and white patches of exudates in a typical DR image. Figure 3 shows the different significant portions of a normal eye image.

\section{Background}

Therapeutic Image Segmentation methods helps to analyze the pathology characteristics by instinctive demarcation of regions in images. Image Segmentation methods isolates substantial regions with analogous features [8]. Image segmentation is process of grouping of pixels with some homogeneous characteristics w.r.t numerous ideologies. Segmentation aims at defining individual distinct regions as connected regions. They are in general categorized into (i) Local and (ii) Global segmentation. Local image segmentation targets a sub portion (kernel or window) of an image and groups the pels based on the selected features. Whereas Global segmentation operates on overall image portion which makes use of estimated parameter values to segment. Image splitting up can be designed using the following three different theoretical perceptions. They include (i) Edge, (ii) boundary, and (iii) Region based methods. In the literature various image segmentation algorithms to detect the lesions in DR images have been explained. Roychowdhury Sohani et.al [9] used a 3 stage algorithms for automated exudate detection and implemented SVM, GMM, ADABOOST, and KNN procedures for classification. Mahendran [10] used morphological and GLCM method for feature extraction, then segmented results are classified with SVM and KNN classifiers. Anushikha Singh et al [11] utilised morphology operators and intensity threshold method to reduce false positives. Mahendran Gandhi [12] proposed an automated exudate detection in diabetic retinopathy through and used SVM classifier to estimate the severity level. Xiwei Zhang et al [13] used random forest algorithm for lesion detection in fundus images. Dr. Ramani R. G. et.al [14], done a fair evaluation of random tree algorithms vs $\mathrm{C} 4.5$ decision tree algorithm and concluded that later method is quite accurate than the former. Jagadish Nayak et.al [15] proposed a comparative evaluation of artificial neural network and Bayesian statistical classifier for to classify fundus pictures. And concluded that neural network gives better results than Bayesian statistical classifier. Luca Giancardo et.al [16] proposed color wavelet based decomposition for lesion detection in Diabetic macular edema. Used the feature like exudate probability, color and wavelet analysis to train the classifier. Agurto. $\mathrm{C}$ et al $[17,18]$ proposed an innovative method called AM and FM for lesion recognition. Osareh et al $[19,20]$ used FCM for coarse segmentation of color retinal images, and the extracted feature from FCM result are used as an input to ANN for classification. Employed a comparative analysis between ANN and SVM classifier and confirmed that later method gives optimum results. Dunn J C [21] reported Fuzzy C means clustering algorithm to cluster data having homogenous attribute. Bezdek J C [22] enhanced FCM. Long Chen et al [23] used multiple kernel distance metric into cost function and demonstrated its significance of their work over traditional FCM algorithm. Abbas. B et. al [24] induced Gaussian spatial info into fuzzy membership function to enhance the significance of the considered pixel from its neighbour pixels in converging the cost function. They proposed that inclusion of spatial info into traditional FCM will in- creases the correlation of pixels located in a region during the convergence. Giri Babu Kande et. al [25] induced spatial weights into FCM to segment hard exudates. A.Sophark et. al [26] used traditional FCM for coarse segmentation of hard exudate images followed with morphological methods for fine tuning. Welfer.D et al [27] allowed L channel in Luv color space to top-hat morphological method to segment fundus retinal images.

This paper is organized as follows: methodology of the proposed method is given in section 3 , results in section 4 followed by conclusions.

\section{Proposed method}

Fuzzy based methods are less complex and more robust. Clustering method groups pixels with common features and accumulates data sets into distinct sets constructed on precise resemblances. In the clustering method, a feature set is extracted from sub portions of an image. Assigning each pixel to its nearest cluster mean is a standard procedure used in clustering methods. Clustering procedures imposes their strength in widespread applications, which includes image processing, classification, pattern recognition, identification and modelling. Single and Dual Kernel induced Spatial FCM is proposed in this work.

Incorporation of Gaussian spatial kernel in KSFCM gives more emphasis in extracting target lesions. Fuzzy clustering methods are more widely used image segmentation methods as they are less complex and robust in computations. Compared to crisp clustering methods they use fuzzy factor which computes the degree of belongingness of pixels with the multiples clusters present in the image. This will be useful to preserve significant data from the original images. Fuzzy based clustering methods reduces inter cluster union by minimizing a proposed distance metric. Usage of kernels in the distance metric improves the computational flexibility and incorporation of spatial information will make the algorithm immune to noise. The approach of the proposed work is as follows:

\subsection{Multi kernel induced spatial fuzzy $C$ Mean cluster- ing algorithm (MKSFCM)}

The image pixels be represented as $\mathrm{x}_{\mathrm{j}}=\left(\mathrm{x}, \mathrm{x}_{2}, \mathrm{x}_{3} \ldots \mathrm{x}_{\mathrm{n}}\right)$ and cluster centres, $c_{i}=\left(c_{1}, c_{2}, c_{3} \ldots \ldots c_{c}\right)$. The cost function to be minimized by the iterative FCM clustering algorithm with kernels induced is given by,

$\mathrm{J}_{\mathrm{KFCM}}=\sum_{\mathrm{i}=1}^{\mathrm{c}} \sum_{\mathrm{j}=1}^{\mathrm{n}} \mathrm{M}_{\mathrm{ij}}^{\mathrm{m}}\left(1-\mathrm{K}\left(\mathrm{x}_{\mathrm{j}}, \mathrm{c}_{\mathrm{i}}\right)\right)$

Here $\mathrm{M}_{\mathrm{ij}}$ is the fuzzy membership value of the jth data point in ith cluster, the parameter $\mathrm{m}$ called fuzzy factor which governs the expanse of fuzziness in the subsequent classification $(m=1$ for crisp clustering) and $\mathrm{K}$ is the induced kernel metric.

The revised cost function of multi kernel FCM with induced Gaussian spatial information is given by,

$\mathrm{J}_{M K S F C M}=\sum_{\mathrm{i}=1}^{\mathrm{c}} \sum_{\mathrm{j}=1}^{\mathrm{n}} \mathrm{M}_{\mathrm{ij}}^{\mathrm{m}}\left(1-\mathrm{K}\left(\mathrm{x}_{\mathrm{j}}, \mathrm{c}_{\mathrm{i}}\right)\right)+\sum_{\mathrm{i}=1}^{\mathrm{c}} \sum_{\mathrm{j}=1}^{\mathrm{n}} \mathrm{h}_{\mathrm{ij}}$

Where $\mathrm{K}_{\mathrm{M}}\left(\mathrm{x}_{\mathrm{j}}, \mathrm{C}_{\mathrm{i}}\right)$ represents a blend of multiple kernels. Equation for two kernels represented as,

$$
\mathrm{K}_{\mathrm{M}}\left(\mathrm{x}_{\mathrm{j}}, \mathrm{C}_{\mathrm{i}}\right)=\pi_{\mathrm{i}=1}^{\mathrm{k}=2} \mathrm{~K}_{\mathrm{i}}\left(\mathrm{x}_{\mathrm{j}}, \mathrm{C}_{\mathrm{i}}\right)
$$

In this work two laplacian kernels defined by $\mathrm{K}_{\mathrm{l}}\left(\mathrm{x}_{\mathrm{j}}, \mathrm{C}_{\mathrm{i}}\right)=$ $\exp \left(\left\|\mathrm{x}_{\mathrm{j}}-\mathrm{c}_{\mathrm{i}}\right\| / \sigma_{\mathrm{k}}\right)$ with standard deviation $\sigma_{1}=100$ and $\sigma_{2}=$ 120 are used. A comparative analysis is done with single and dual kernel induced Spatial FCM algorithm. Hence the proposed algorithm is noted in short form as L1KSFCM (SFCM with single kernel) and L2KSFCM (SFCM with dual kernels). 
Here $\mathrm{k}$ is the number of kernels and $\mathrm{h}_{\mathrm{ij}}$ is the added spatial constraint which is defined as

$$
\mathrm{h}_{\mathrm{ij}}=\sum_{\mathrm{k} \in \mathrm{NB}_{\mathrm{x}_{\mathrm{j}}}} \sum_{\mathrm{l} \in \mathrm{NB}_{\mathrm{x}_{\mathrm{j}}}} \frac{1}{2 \pi \sigma^{2}} \mathrm{e}^{\frac{\mathrm{k}^{2}+\mathrm{l}^{2}}{2 \sigma^{2}}} \mathrm{M}_{\mathrm{k}, \mathrm{l}}
$$

$\mathrm{h}_{\mathrm{ij}}$ is the spatial function which operates on $\mathrm{x}_{\mathrm{j}}$ and its neighbors defined in a sub image sub portion $\mathrm{NB}_{\mathrm{x}_{\mathrm{j}}}(5 \times 5$ matrix $)$. This signifies probability of degree of belongingness of the pixel $x_{j}$ to the $\mathrm{i}^{\text {th }}$ cluster in the image with $\mathrm{x}_{\mathrm{j}}$ as it center.

The cluster centers $C_{i}$ and the membership function $M_{i j}$ are updated at each iteration inorder to optimize the cost function. The respective equations of $\mathrm{M}_{\mathrm{ij}}$ and $\mathrm{C}_{\mathrm{i}}$ are defined as follows,

$$
\begin{gathered}
\mathrm{M}_{\mathrm{ij}}=\frac{\left(1-\mathrm{K}_{\mathrm{M}}\left(\mathrm{x}_{\mathrm{j}}, \mathrm{C}_{\mathrm{i}}\right)\right)^{\frac{-1}{\mathrm{~m}-1}}}{\sum_{\mathrm{i}=1}^{\mathrm{c}}\left(1-\mathrm{K}_{\mathrm{M}}\left(\mathrm{x}_{\mathrm{j}}, \mathrm{C}_{\mathrm{i}}\right)\right)^{\frac{-1}{\mathrm{~m}-1}}} \\
\mathrm{C}_{\mathrm{i}}=\frac{\sum_{\mathrm{j}=1}^{\mathrm{n}} \mathrm{M}_{\mathrm{ij}}{ }^{\mathrm{m}} \mathrm{K}_{\mathrm{M}}\left(\mathrm{x}_{\mathrm{j}}, \mathrm{C}_{\mathrm{i}}\right) \mathrm{x}_{\mathrm{j}}}{\sum_{\mathrm{j}=1}^{\mathrm{n}} \mathrm{M}_{\mathrm{ij}}^{\mathrm{m}} \mathrm{K}_{\mathrm{M}}\left(\mathrm{c}_{\mathrm{j}}, \mathrm{C}_{\mathrm{i}}\right)}
\end{gathered}
$$

Where $\mathrm{i}=1,2,3 \ldots, \mathrm{C}$.

The modified membership function with included spatial function $\mathrm{h}_{\mathrm{ij}}$ is given as

$$
\mathrm{M}_{\mathrm{ij}}^{\prime}=\frac{\mathrm{M}_{\mathrm{ij}}^{\mathrm{m}} \mathrm{h}_{\mathrm{ij}}}{\sum_{\mathrm{k}=1}^{\mathrm{c}} \mathrm{M}_{\mathrm{ij}}^{\mathrm{m}} \mathrm{h}_{\mathrm{ij}}}
$$

The modified membership function is updated iteratively and is further appended into eq (6) in each iteration.

Algorithm of the proposed work:

1) Fix the number of clusters ' $C$ ' and randomly initialize the cluster centers $\mathrm{C}_{\mathrm{i}}$

2) Update membership matrix $M_{i j}$ using the equation (5)

3) Update cluster centers $C_{i}$ using the equation (6)

4) Update membership matrix $\mathrm{M}_{\mathrm{ij}}$ using the equation (7)

5) Update Objective function $\mathrm{J}_{\mathrm{MKSFCM}}$ using equation (2)

If $\left|J^{\mathrm{t}}-\mathrm{J}^{\mathrm{t}-1}\right|<\varepsilon$ stop the iteration process otherwise repeat the steps 2 to 4 Where $\varepsilon=0.0001$

1.1 Simulation of proposed work using Raspberry pi Model B:

The proposed method implemented in Raspberry pi 3 Model B with 1GB RAM, quad core 1.2 Ghz processor kit. Raspbian Jessie OS and Octave 4.0 application with Image tool box is installed. The implementation of the proposed work on Raspberry pi kit is shown in Figure 4.

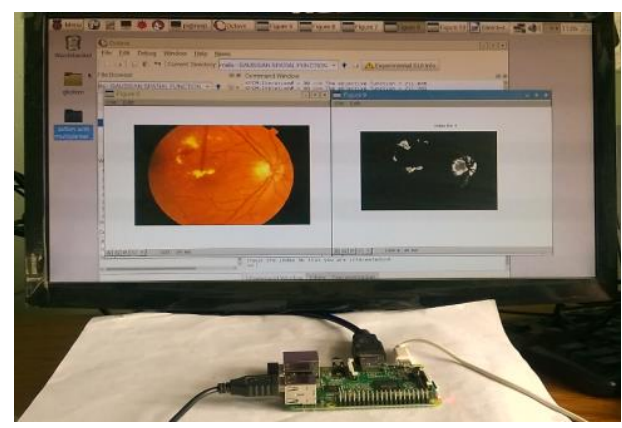

Fig. 4: Implementation of Proposed Work Using Raspberry Pi 3 Model B.

\section{Experimental results}

Green from the retina images affected by DR pathos are used as an input to the proposed algorithm, since it carries high contrast value. This component is applied to median filter in the preprocessing step to suppress any inherent noise present in the images. Then it is applied to the proposed algorithms for segmentation. The optic disc was selectively segregated to avoid unnecessary false alarms.

The performance of the proposed method is statistically (Sensitivity and Specificity) evaluated using clinician ground truth images. The parameters Sensitivity and Specificity which are given by,

Sensitivity $=\frac{\mathrm{TP}}{\mathrm{TP}+\mathrm{FN}}$

Specificity $=\frac{\mathrm{TN}}{\mathrm{TN}+\mathrm{FP}}$

TP (True Positive): correctly classified Exudate pixels

TN (True Negative): correctly classified non-exudate pixels

FP (False Positive): incorrectly classified Exudate pixels

FN (False Negative): incorrectly classified non-Exudate pixels

A total of 40 images taken from public databases Diaretdb0 [28],

Diaretdb1 [29], American Society of Retina Specialists [30], eophtha: A Color Fundus Image Database [31], High-Resolution Fundus (HRF) Image Database [32] and one private database provided by Suthrama Eye Hospital, Madanapalle are used in this work.

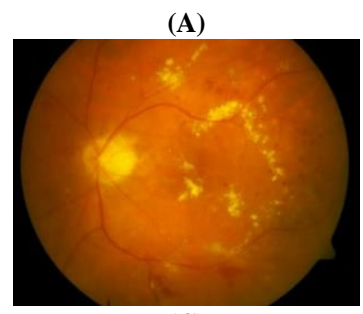

(C)

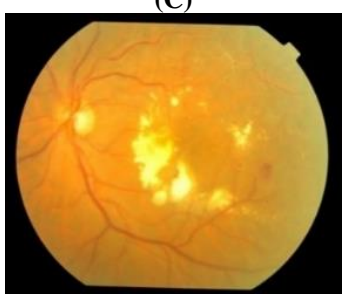

(E)

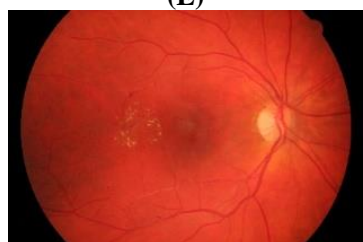

Fig. 5: Typical DR Images with Exudates: (A) Image Courtesy: Diaretdb0, (B) Image Courtesy: Diaretdb1, (C) Image Courtesy: American Society of Retina Specialists, (D) Image Courtesy: E-Ophtha (2013) (E) Image Courtesy: High-Resolution Fundus (HRF) Databases (F) Image Courtesy: Suthrama Eye Hospital, Madanapalle

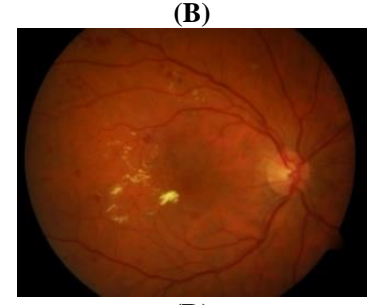

(D)

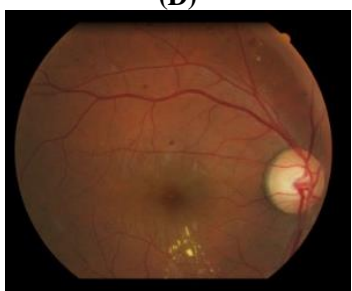

(F)

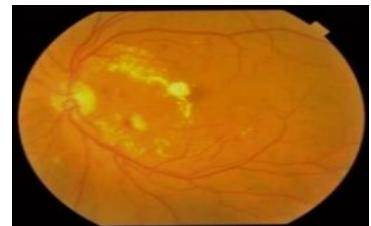




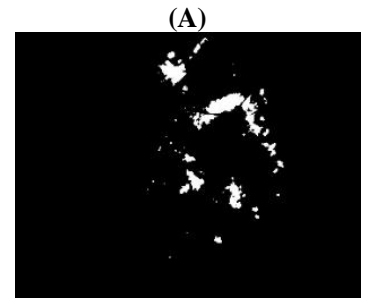

(C)

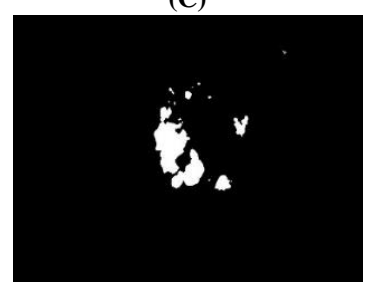

(E)

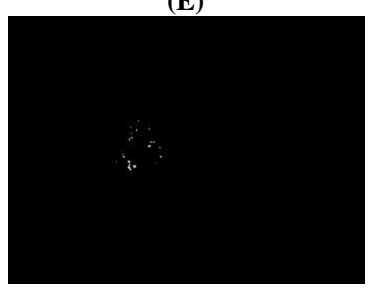

Fig. 6: A-F Are Respective L1KSFCM Output Images of Figure 5: A-F

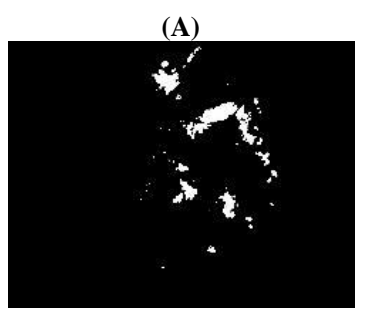

(C)

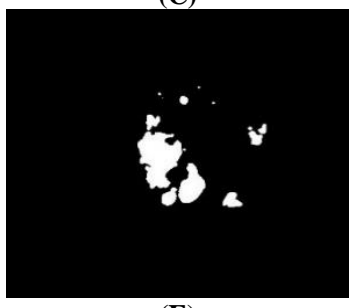

(E)

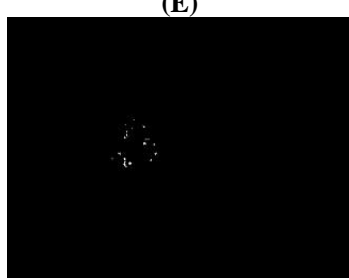

Fig. 7: A-F are Respective L2KSFCM Output Images of Figure 5: A-F.

Figure 5 shows the typical DR images taken from multiple sources. Figure $6 \&$ Figure 7 shows the respective output images of figure 5 when applied to L1KSFCM and L2KSFCM methods. The fine details of lesions are quite evitable in the results.

Sensitivity and Specificity are calculated using the clinician ground truth images. The Quantitative evaluation of the proposed method with the existing methods are shown in table 1 .

Table 1: Statistical Evaluation

\begin{tabular}{lll}
\hline Author & Sensitivity & Specificity \\
\hline Giribabu Kande et al (25) & 86 & 98 \\
Sopharpak et al (26) & 87.28 & 99.24 \\
Welfer et al (27) & 70.48 & 98.84 \\
Proposed methods & & \\
L1KSFCM & 87.76 & 99.10 \\
L2KSFCM & 91.38 & 99.38 \\
\hline
\end{tabular}

The image details like file size, dimensions and resolution of four DR images represented in the results are shown in the table 2 . The elapsed time to run (for 50 iterations) the proposed algorithms using Intel i5 $52002.20 \mathrm{GHz}$ CPU vs Raspberry pi 3 Model B with 1GB RAM, quad core $1.2 \mathrm{GHz}$ CPU are tabulated in table 3 and table 4:

Table 2: DR Images File Characteristics

\begin{tabular}{|c|c|c|c|}
\hline Original image & File Size (MB) & Dimension & File type \\
\hline Figure $5(\mathrm{a})$ & $1.26 \mathrm{MB}$ & $1500 * 1152$ & png \\
\hline Figure 5(b) & $1.57 \mathrm{MB}$ & $1500 * 1152$ & png \\
\hline Figure 5(c) & $112 \mathrm{~KB}$ & $643 * 427$ & jpg \\
\hline Figure $5(d)$ & $553 \mathrm{~KB}$ & $2544 * 1696$ & jpg \\
\hline Figure 5(e) & $906 \mathrm{~KB}$ & $3504 * 2336$ & jpg \\
\hline Figure 5(f) & $289 \mathrm{~KB}$ & $2144 * 1424$ & jpg \\
\hline \multicolumn{4}{|c|}{ Table 3: Elapsed Time in Seconds } \\
\hline L1KSFCM & \multicolumn{2}{|c|}{ Intel Core i5 } & $\begin{array}{l}\text { Raspberry Pi } \\
\text { Model B }\end{array}$ \\
\hline Original image & Matlab R2015a & Octave 4.0 & Octave 4.0 \\
\hline Figure $5(\mathrm{a})$ & 133.635 & 140.31 & 592.2 \\
\hline Figure 5(b) & 148.75 & 178.64 & 755.64 \\
\hline Figure 5(c) & 28.418 & 36.94 & 158.84 \\
\hline Figure $5(d)$ & 304.8404 & 373.92 & 1577.94 \\
\hline Figure 5(e) & 854.99 & 949.64 & 4016.97 \\
\hline Figure 5(f) & 150.017 & 184.82 & 784.56 \\
\hline \multicolumn{4}{|c|}{ Table 4: Elapsed Time in Seconds } \\
\hline L2KSFCM & \multicolumn{2}{|c|}{ Intel Core i5 } & $\begin{array}{l}\text { Raspberry Pi } \\
\text { Model B }\end{array}$ \\
\hline Original image & Matlab R2015a & Octave 4.0 & Octave 4.0 \\
\hline Figure 5(a) & 143.884 & 189.92 & 782.47 \\
\hline Figure 5(b) & 167.428 & 208.75 & 859.04 \\
\hline Figure 5(c) & 31.808 & 38.634 & 163.70 \\
\hline Figure 5(d) & 324.833 & 405.972 & 1717.38 \\
\hline Figure 5(e) & 1017.59 & 1241.45 & 5245.2 \\
\hline Figure 5(f) & 195.43 & 253.52 & 1031.68 \\
\hline
\end{tabular}

From these tables it can be observed that the runtime of the algorithms is dependent on the resolution of the images.

\section{Conclusion}

From the result analysis it is evident that the proposed works L1KSFCM and L2KSFCM algorithm extracted fine details of exudates from the DR fund scope images and achieved good sensitivity and specificity. The simulation is carried out using PC and Raspberry pi 3 Model B. Further the merging features of this method can be improved with implementation of adaptive weighted membership function.

\section{Acknowledgement}

The authors thank Dr.N.Praveen Kanth Reddy, Ophthalmologist, Suthrama Eye hospital, Madanapalle for his help to carry out this work.

\section{References}

[1] "Diabetic retinopathy: what you should know", National Eye Institute, National Institute of Health, NIH Publication No. 15-2171, 2017.

[2] Dharmalingam M. (2003) "Diabetic retinopathy-risk factors and strategies in prevention", Laser, vol. 51, pp. 77.

[3] Stratton I. M., Aldington S. J., Taylor D. J. et al. (2012) "A Simple Risk Stratification for Time to Development of Sight-Threatening Diabetic Retinopathy. Diabetes Care", pp. 1-6, November 12.

[4] Neeti Gupta, Rohit Gupta (Jan. - Mar. 2015), "Diabetic Retinopathy - An Update", JIMSA Vol. 28 No. 1.

[5] Akara Sopharak, Bunyarit Uyyanonvara, Sarah Barman, Thomas H. Williamson (2008)," Automatic detection of diabetic retinopathy exudates from non-dilated retinal images using mathematical morphology methods", Computerized Medical Imaging and Graphics 
32

$\mathrm{pp}$

https://doi.org/10.1016/j.compmedimag.2008.08.009.

[6] C.I. Sanchez, R. Hornero, M.I. Lopez, J. Poza. 2004 "Retinal Image Analysis to Detect and Quantify Lesions Associated with Diabetic Retinopathy", Proc. 26th IEEE Annual International Conf. on Engineering in Medicine and Biology Society (EMBC), 3, $1624-$ 1627. https://doi.org/10.1109/IEMBS.2004.1403492.

[7] Selvathi D, N.B.Prakash, and Neethi Balagopal. 2012 "Automated Detection of Diabetic Retinopathy for Early Diagnosis using Feature Extraction and Support Vector Machine", International Journal of Emerging Technology and Advanced Engineering (ISSN 22502459, Volume 2, Issue 11.

[8] Jayaraman S, Esakkirajan S. and Veera Kumar T. (2013), "Digital Image processing", McGraw Hill Education (India) pvt ltd.

[9] Sohini Roychowdhury, Dara D. Koozekanani, and Keshab K. Parhi (September-2014), "DREAM: Diabetic Retinopathy Analysis Using Machine Learning", IEEE Journal of Biomedical and Health Information, VOL. 18 pp. 1717 - 1728, No. 5.

[10] Mahendran Gandhi, and Dr. R. Dhanasekaran (2013), "Diagnosis of Diabetic Retinopathy Using Morphological Process and SVM Classifier", Communications and Signal Processing (1CCSP), International Conference on pp. $873-877$.

[11] Anushikha Singh, Namita Sengar, Malay Kishore Dutta, Kamil Riha, Jiri Minar (2015), "Automatic exudates detection in fundus image using intensity thresholding and morphology", Seventh ICUMT, $\quad$ IEEE, $\quad$ p. $330 \quad 334$ https://doi.org/10.1109/ICUMT.2015.7382452.

[12] Mahendran Gandhi, and Dr. R. Dhanasekaran (April 2013), "Diagnosis of Diabetic Retinopathy Using Morphological Process and SVM Classifier". International conference on Communication and Signal Processing, Melmaruvathur, India, pp.952-956. https://doi.org/10.1109/iccsp.2013.6577181.

[13] Xiwei Zhang, Guillaume Thibault, Etienne Decencière, Beatriz Marcotegui (2014), "Exudate Detection in Color Retinal Images for Mass Screening of Diabetic Retinopathy", Medical Image Analysis, Volume 18(7), P. 1026-1043, ELSEVIER. https://doi.org/10.1016/j.media.2014.05.004.

[14] Dr.R.Geetha Ramanil et al (Dec. 2012), "Data Mining Method of Evaluating Classifier Prediction Accuracy in Retinal Data", Computational Intelligence \& Computing Research (ICCIC), 2012 IEEE International Conference on 18-20 pp.1 - 4.

[15] Jagadish Nayak et.al. (April 2008), “Automated Identification of Diabetic Retinopathy Stages Using Digital Fundus Images", Journal of Medical Systems, Volume 32, Issue 2, pp 107-115. https://doi.org/10.1007/s10916-007-9113-9.

[16] Luca Giancardo, Fabrice Meriaudeau, Thomas P. Karnowski, et.al (January 2012), "Exudate-based diabetic macular edema detection in fundus images using publicly available datasets", Medical Image Analysis: Volume 16, Issue I, Pages 216-226. https://doi.org/10.1016/j.media.2011.07.004.

[17] Agurto .C, et.al. (2010 Feb), "Multiscale AM-FM methods for diabetic retinopathy lesion detection", IEEE Trans Med Imaging.; 29(2):502-12. doi: 10.1 109/TM1.2009.2037 146

[18] Agurto.C, et.al. (Feb. 2010), "Multiscale am-fin methods for diabetic retinopathy lesion detection", Medical Imaging. IEEE Transactions, vol. 29, No. 2, pp. $502 \quad-512$ https://doi.org/10.1109/TMI.2009.2037146.

[19] Osareh A, Mirmehdi M, Thomas B, Markham R (2001), "Automatic recognition of exudative maculopathy using fuzzy c-means clustering and neural networks", Medical Image Understanding Analysis, BMVA Press, UK, pp. 49-52.

[20] Osareh, B. Shadgar, and R. Markham (2009), “A ComputationalIntelligence-Based Approach for Detection of Exudates in Diabetic Retinopathy Images", Information Technology in Biomedicine, IEEE Transactions on, vol. 13, pp. 535-545. https://doi.org/10.1109/TITB.2008.2007493.

[21] Dunn J C, J.Cybernet (March, 1973), "A fuzzy relative of the ISODTATA process and its use in detecting compact well separated clusters", pp 32-57.

[22] Bezdek J C (1981), "Pattern Recognition with Fuzzy Objective Function Algorithms", New York: Plenum Press. https://doi.org/10.1007/978-1-4757-0450-1.

[23] Long Chen, C L Philip Chen, Mingzhu Lu .A (2011), "MultipleKernel Fuzzy C-Means Algorithm for Image Segmentation”. IEEE Systems, Man, and Cybernetics Society, p. 1263 - 1274. https://doi.org/10.1109/TSMCB.2011.2124455.

[24] Abbas Biniaz, Ataollah Abbassi, Mousa Shamsi, Afshin Ebrahimi. (2012), "Fuzzy c-means clustering based on Gaussian spatial information for brain MR image segmentation", IEEE 19th Iranian
Conference of on Biomedical Engineering (ICBME), p.154 - 158. https://doi.org/10.1109/ICBME.2012.6519676.

[25] Giri Babu Kande, P.Venkata Subbaiah, T.Satya Savithri 3(2008), "Segmentation of Exudates and Optic Disc in Retinal Images". Sixth Indian Conference on Computer Vision, Graphics \& Image Processing,

[26] Akara Sopharak , Bunyarit Uyyanonvara and Sarah Barman (2009), "Automatic Exudate Detection from Non-dilated Diabetic Retinopathy Retinal Images Using Fuzzy C-means Clustering”, 9, 2148-2161; https://doi.org/10.3390/s90302148.

[27] Daniel Welfer, Jacob Scharcanski Diane Ruschel Marinho (2010), "A coarse-to-fine strategy for automatically detecting exudates in color eye fundus images", Computerized Medical Imaging and Graphics 34, pp 228-235 https://doi.org/10.1016/j.compmedimag.2009.10.001.

[28] Kauppi. T, Kalesnykiene T, Kamarainen J.K, Lensu L, Sorri I, Uusitalo H. Kalviainen H and Pietila. J (2006) "Diaretdb0: Evaluation database and methodology for diabetic retinopathy algorithms", Technical report Lappeenranta University of Technology Finland.

[29] Kauppi, T., Kalesnykiene, V., Kamarainen, J.K., Lensu, L., Sorri, I., Raninen A., Voutilainen R., Uusitalo, H., Kälviäinen, H., Pietilä, J., "DIARETDB1 diabetic retinopathy database and evaluation protocol", Proc of the 11th Conf. on Medical Image Understanding and Analysis (Aberystwyth, Wales, 2007). https://doi.org/10.5244/C.21.15.

[30] ASRS: Advocating for You and Your Patients in 2017, Retina TimesSpring 2017, Vol. 35, No. 1, Issue 68.

[31] Decencière E, et al. TeleOphta: Machine learning and image processing methods for teleophthalmology. IRBM, (2013), https://doi.org/10.1016/j.irbm.2013.01.010.

[32] Budai, Attila; Bock, Rüdiger; Maier, Andreas; Hornegger, Joachim; Michelson, Georg (2013), "Robust Vessel Segmentation in Fundus Images, International Journal of Biomedical Imaging”, vol. 2013. https://doi.org/10.1155/2013/154860. 